\title{
BCG-Impfung: kein Einfluß auf das Atopie-Risiko
}

\section{Eine frühzeitige BCG-Impfung von Kindern mit erblicher Atopie- Belastung scheint das Risiko, bis zum Schulalter eine allergische Erkankung zu entwickeln, weder zu senken noch zu erhöhen.}

D ie Häufigkeit von atopischen Erkrankungen nimmt in den Industrienationen seit Jahren zu. Der Anteil von Kindern mit Asthma, atopischem Ekzem, Heuschnupfen oder Nahrungsmittelallergien liegt derzeit bereits bei über $30 \%$. Die Ursachen dieser Entwicklung sind nach wie vor unklar. Diskutiert wird unter anderem ein Zusammenhang mit der Abnahme bestimmter Infektionskrankheiten, aber auch mit Änderungen in den Impfprogrammen. So hatte eine japanische Arbeitsgruppe Anfang des Jahres Hinweise für einen präventiven Effekt einer BCG-Impfung geliefert.

Im Rahmen einer retrospektiven Studie am Karolinska-Institut in Stockholm wurde jetzt ebenfalls der Frage nachgegangen, ob die BCGImpfung gegen Tuberkulose die Entstehung von Atopien beeinflußt. Hier$\mathrm{zu}$ untersuchten die schwedischen Wissenschaftler 216, aus AllergikerFamilien stammende Kinder, die im Alter unter 6 Monaten in den Jahren zwischen 1989 und 1992 eine BCGImpfung erhalten hatten. Als Vergleichsgruppe dienten 358 gleichaltrige Kinder, die nicht geimpft worden waren.

Bei allen Kindern wurde in der Zeit zwischen 1995 und 1996 nach Anzeichen von atopischen Erkrankungen gefahndet. Überdies wurden Allergen-spezifische IgE-Antikörper untersucht und Prick-Tests durchgeführt.

\section{Keine Unterschiede zwischen Impf- lingen und Kontrollgruppe}

Die Ergebnisse: 77 Kinder der BCG-Gruppe (36\%) und 145 Kinder der Kontrollgruppe (41\%) zeigten klinische Zeichen einer atopischen Erkrankung oder eine positive AtopieAnamnese. Der Prick-Test fiel bei
$12 \%$ der geimpften Kinder positiv aus, in der Kontrollgruppe bei $10 \% .31 \%$ der Impflinge und 27\% der Kinder in der Kontrollgruppe wiesen zirkulierende Allergen-spezifische IgE-Antikörper auf. Bei jeweils zwei Drittel der Kinder in beiden Gruppen wurden auch bei den Eltern serologische Hinweise auf eine Atopie gefunden. Andere Risikofaktoren für allergische Erkankungen waren in beiden Gruppen gleich häufig.

Insgesamt schien die BCG-Impfung keinen Einfluß auf das Auftreten einer Allergie zu haben. (mo)

Quelle: J.S. Alm, et al.: Early BCG vaccination and develop ment of atopy. Lancet 1997; 350: 400-403.

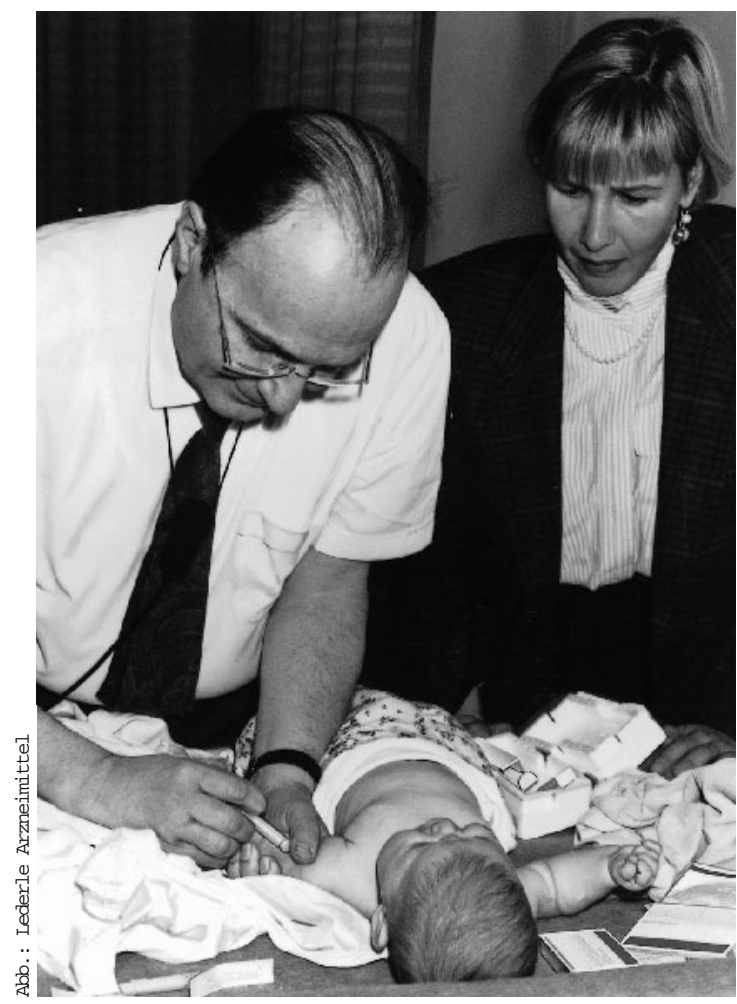

BCG-Impfung: nicht ausreichend immunogen für einen TH2/TH1-Switch?

\section{Kommentar}

Die Zunahme der atopischen Erkrankungen in der industrialisierten Welt wird zum Teil mit der Abnahme der Infektionskrankheiten und der Änderung im Impfverhalten erklärt. Das simplifizierte T-Helfer-Zell-Modell (TH1/TH2-Dichotomie) stützt diese Hypothese. Demnach schützt ein zur TH1-Immunantwort führender Stimulus möglicherweise vor der durch die TH2-Antwort bedingte Allergie.

Dieses in Mäusen generierte TH1/TH2-Modell ist die Grundlage verschiedener Studien. So beschrieben Shirakawa et al. Anfang des Jahres in Science den inversen Zusammenhang zwischen positivem Tuberkulinhauttest und atopischer Erkrankung bei japanischen Schulkindern. $\mathrm{Ob}$ die Tuberkulinreaktivität durch eine BCG-Impfung, durch eine primäre Tuberkulose-Infektion oder durch Umweltmykobakterien verursacht war, blieb jedoch unklar. Hingegen konnte in der vorliegenden, sorgfältig durchgeführten schwedischen FallKontroll-Studie kein Effekt einer BCG-Impfung auf die atopische Manifestation bei Kleinkindern atopischer Hochrisikofamilien gezeigt werden.

Im Tiermodell ließ sich zwar eine Änderung des Zytokinmusters vom TH2- zum TH1-Typ hin durch eine intravenöse BCG-Impfung als TH1-Stimulus induzieren. $\mathrm{Ob}$ und $\mathrm{zu}$ welchem Zeitpunkt aber eine intrakutane BCG-Impfung bei Kindern mit den zur Zeit verwendeten BCG-Impfstämmen immunogen genug ist, außer einer Tuberkulin-Reaktivität auch einen TH2/TH1-Switch bei prädisponierten $\mathrm{TH} 2-\mathrm{Re}-$ spondern zu induzieren, bleibt die zu klärende Frage.

Korrespondenzadresse:

Dr. med. Rainer Kehrt, Medizinische Fakultät der Humboldt-Universität zu Berlin, Virchow-Klinikum, Abteilung Pädiatrie, Augustenburger Platz 1, 13353 Berlin. 